\title{
Evaluation of qualitative attributes of papaya leather
}

\author{
Devendra Kumar and R.N. Shukla
}

Received : 26.01.2018; Revised : 13.02.2018; Accepted : 22.02.2018

See end of the Paper for authors' affiliation

Correspondence to :

Devendra Kumar

B.S. Dr. B.R.A. College of Agricultural Engineering and Technology (C.S.A.U.A.T.)

Etawah (U.P.) India

Email : devendrachaturvedi 2008@gmail.com
- ABSTRACT : India is major producer of papaya after Brazil and Indonesia. Papaya contains the digestive enzyme papain and valuable for aiding digestion. The antioxidant nutrients found in papaya including vitamin-C, vitamin-E and beta carotene. Papaya is easily digestible and prevents constipation. Fruit leather is ready to eat, semi- moist food with soft gel like texture obtained by dehydration of fruit purees into leathery sheets. Study of quality attributes like physio-chemical, sensory and microbiological properties of fruit leather resulted better and acceptable products. Experiments were conducted to investigate the effect of various sweeteners and packaging materials on physico-chemical, microbiological properties and sensory characteristics of fresh as well as stored papaya leather. The citric acid levels of 0.5 per cent, 0.75 per cent and 1.0 per cent were used for sweeteners as sugar, sugar $_{75}+$ jaggery $_{25}$, sugar $_{50}+$ jaggery $_{50}$, sugar $_{25}+$ jaggery $_{75}$ and jaggery. After preparation of papaya leather, the finished products were packed in two packaging material viz., LDPE and HDPE and stored at room temperature for quality evaluation at 15 days interval upto 90 days. The study revealed that the moisture content increased with citric acid levels in case of all different sweeteners. The values of moisture content were found to have decrease after 15, 30, 45, 60 and 90 days of storage. The data showed that the samples packed in LDPE more decreases as compared to HDPE. The TSS was found to be higher for fresh samples prepared by sugar as sweeteners at all levels of citric acid. TSS of samples packed in HDPE were found to be higher than LDPE at the same level of citric acid. $\mathrm{pH}$ of samples after 90 days of storage periods prepared by sugar as a sweeteners were found to be lower than that of $\operatorname{sugar}_{75}+$ jaggery $_{25}, \operatorname{sugar}_{50}+$ jaggery $_{50}$, sugar $_{25}+$ jaggery $_{75}$ and jaggery at all levels of citric acid. Data obtained for browning index after 15, $30,45,60,75$ and 90 days of storage indicated that in case of all samples, the values increased for all different sweeteners. The study revealed that vitamin-C content of fresh papaya leather sample decreased with increase in citric acid levels in case of all sweeteners. In microbiological studies, the yeast and mold count and total plate count were found safe for consumption after 90 days of storage. Samples prepared by sugar as a sweeteners exhibited the highest overall sensory scores 7.64 and 7.60 for samples packed in HDPE and LDPE, respectively after 90 days of storage periods at the level of 0.75 per cent citric acid. It concluded that sugar as sweeteners gave better products after 90 days of storage followed by others at the level of 0.75 per cent citric acid. The HDPE was found suitable packaging material for storage of papaya leather.

- KEY WORDS : Papaya, Sweetener, Physico-chemical, Microbiological, Sensory

-HOW TO CITE THIS PAPER : Kumar, Devendra and Shukla, R.N. (2018). Evaluation of qualitative attributes of papaya leather. Internat. J. Agric. Engg., 11(1) : 84-89, DOI: 10.15740/HAS/IJAE/11.1/ 84-89. 